\title{
Variable expression observed in a Korean family with Townes-Brocks syndrome caused by a SALL1 mutation
}

\author{
Yeon Jeong Seo', Ko Eun Lee', Jung Min Ko ${ }^{1, *}$, Gu-Hwan Kim², and Han-Wook Yoo ${ }^{2}$ \\ ${ }^{1}$ Department of Pediatrics, Seoul National University Children's Hospital, Seoul, Korea \\ ${ }^{2}$ Medical Genetics Center, Asan Medical Center Children's Hospital, University of Ulsan College of Medicine, Seoul, Korea
}

\begin{abstract}
Townes-Brocks syndrome (TBS) is a rare genetic disorder characterized by the classic triad of congenital anomalies of the anus, thumbs, and ears, with variable expressivity. Additionally, renal malformations, cardiac anomalies, and endocrine and eye abnormalities can accompany TBS, although less frequently. TBS is inherited in an autosomal dominant fashion; however, about $50 \%$ of patients have a family history of TBS and the remaining $50 \%$ have de novo mutations. SALL1, located on chromosome 16q12.1, is the only causative gene of TBS. SALL1 acts as a transcription factor and may play an important role in inducing the anomalies during embryogenesis. Clinical features of TBS overlap with those of other multiple anomaly syndromes, such as VACTERL syndrome, Baller-Gerold syndrome, Goldenhar syndrome, cat eye syndrome, and Holt-Oram syndrome. Consequently, there are some difficulties in differential diagnosis based on clinical manifestations. Herein, we report a Korean family with two generations of TBS that was diagnosed based on physical examination findings and medical history. Although the same mutation in SALL1 was identified in both the mother and the son, they displayed different clinical manifestations, suggesting a phenotypic diversity of TBS.
\end{abstract}

Key words: Townes-Brocks syndrome, SALL1 protein, human, Imperforate anus, Polydactyly, Sensorineural hearing loss.

\section{Introduction}

Townes-Brocks syndrome (TBS) is a rare genetic disorder caused by mutations in SALL1, located on chromosome 16q12.1 [1]. The syndrome can be inherited in an autosomal dominant manner [2]. The frequency of TBS has been estimated to be 1 in 250,000 [3], but the exact prevalence of TBS is uncertain, because the features of TBS overlap with those seen in other syndromes such as VACTERL syndrome. Multiple systemic malformations are accompanied by phenotypic variability.
Commonly affected anomalies are anorectal malformations (imperforate anus, anteriorly placed anus, anal stenosis), hand malformations (preaxial polydactyly, triphalangeal thumb, bifid thumb), external ear malformations (microtia, "satyr" or "lop" ear, preauricular tag or pits) with sensorineural hearing loss, and renal malformations. In addition, intellectual disability, learning problems, and cardiac anomalies have been reported in TBS [46].

Cytogenetic findings provided the first indication of the causative gene for TBS, and SALL 1 was mapped to chromosome

\footnotetext{
Received: 1 June 2015, Revised: 9 June 2015, Accepted: 10 June 2015, Published: 30 June 2015

*Corresponding author: Jung Min Ko, M.D., Ph.D.

Department of Pediatrics, Seoul National University Children's Hospital, 101 Daehak-ro, Jongno-gu, Seoul 110-769, Korea.

Tel: +82-2-2072-3570, Fax: +82-2-743-3455, E-mail: jmko@snu.ac.kr

Conflict of interest: We declare that we do not have any conflicts of interests.

(c) This is an open-access article distributed under the terms of the Creative Commons Attribution Non-Commercial License (http://creativecommons.org/licenses/by-nc/4.0/) which permits unrestricted non-commercial use, distribution, and reproduction in any medium, provided the original work is properly cited.

(c) Copyright 2015 by the Korean Society of Medical Genetics

www.e-kjgm.org
} 
$16 q 12.1$ as the causative gene [7]. SALL1 is a human homolog of SAL, the developmental regulator of Drosophila melanogaster, and SALL 1 encodes zinc finger domain proteins, which are thought to act as a transcription factor [8].

Because of its rarity, there have been only three reports of TBS in Korea [9-11]. Among them, TBS has been confirmed in only one patient by SALL1 analysis to date [11]. Here, we report a Korean family with TBS in the mother and the son, who have the same mutation in SALL 1 and yet show different clinical manifestations.

\section{Case}

\section{Patient 1 (son)}

As a 10-month-old male infant, patient 1 visited the Seoul National University Children's Hospital outpatient clinic to exclude VACTERL syndrome. He was delivered by Cesarean section because of fetal deceleration at the 40th week of gestation with a birth weight of 2,660 $\mathrm{g}(<3 \mathrm{rd}$ percentile). He was the first child of his parents. With the exception of his mother (patient 2), the other family members did not present with any congenital anomalies (Fig. 1). At birth, he had a low type imperforate anus with a penoscrotal fistula, preaxial polydactyly in both hands and triphalangeal thumbs, and dysplastic external ears (Fig. 2A and B). He underwent anoplasty when he was two days old.
Chromosomal analysis of his peripheral blood revealed karyotype $46, X Y$. Echocardiography, performed at nine days of age to evaluate multiple anomalies, revealed a muscular ventricular septal defect (VSD) and patent foramen ovale. Bilateral abnormal results from an automated auditory brainstem response screening test were noted, but no further evaluation had been performed before his visit to our hospital. There was no abnormal result in a neonatal screening test for inherited metabolic diseases.

The patient was referred to the Seoul National University Children's Hospital for further evaluation and management of VACTERL syndrome at the age of eight months. Physical examination during his visit revealed that his height was 73.7 $\mathrm{cm}$ (50th-75th percentile), weight was $9.2 \mathrm{~kg}$ (25th-50th percentile), and head circumference was $44.5 \mathrm{~cm}$ (25th-50th percentile). His right ear was microtia grade I and the superior helix was overfolded (Fig. 2B), and the left ear was a lop ear. He had bilateral supernumerary thumbs and radiography showed preaxial polydactyly with triphalangeal thumbs (Fig. $2 \mathrm{~A}$ and $\mathrm{B})$. Follow-up echocardiography revealed that the VSD had spontaneously closed. There were no abnormalities of his intra-abdominal organs, including the genitourinary system, as determined from abdominal ultrasonography. Ophthalmologic examination showed bilateral coloboma of the irises and no abnormal findings of the fundi. Audiometric examinations
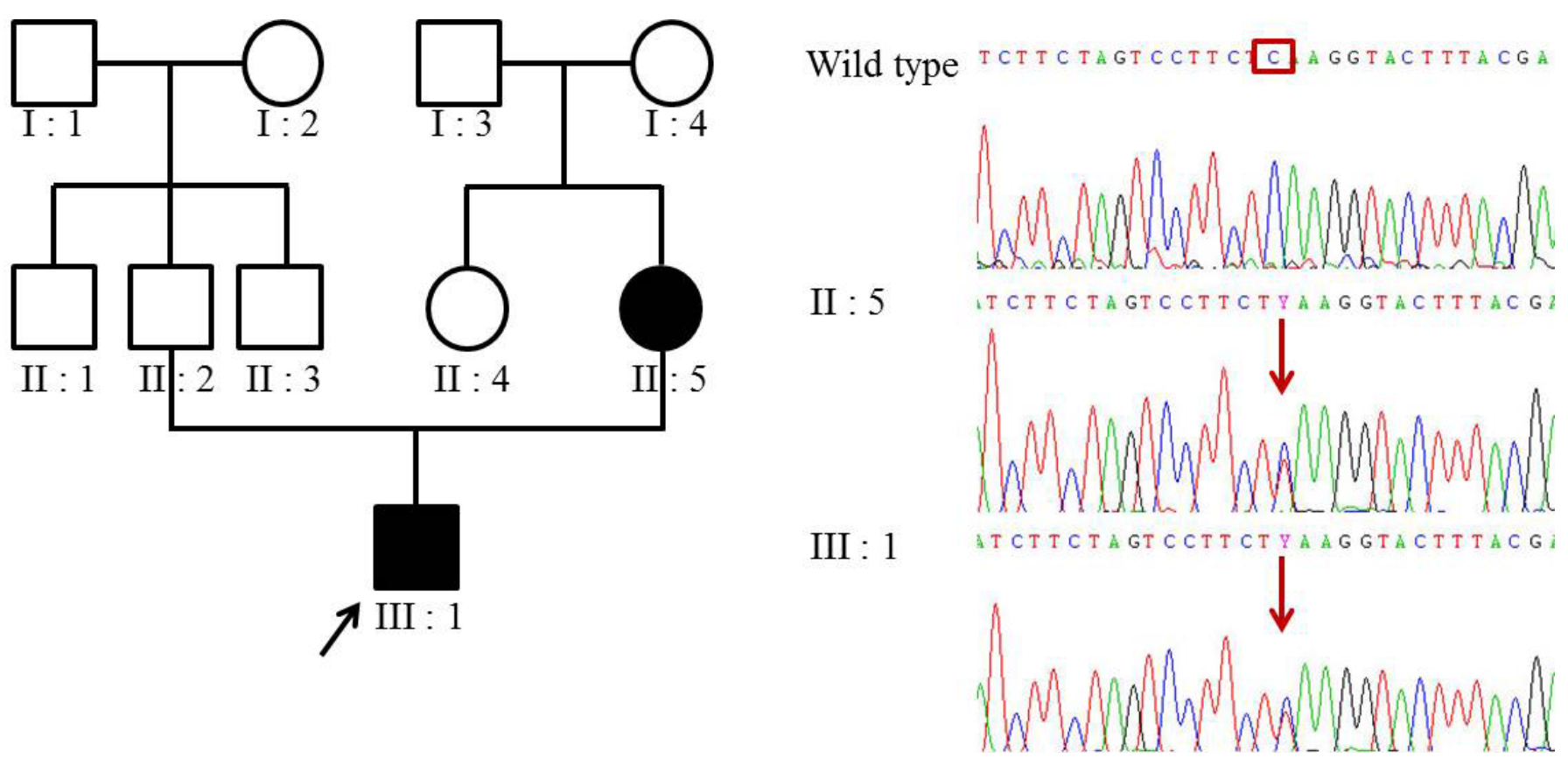

Fig. 1. Pedigree and sequence electropherogram. Partial sequences of $S A L L 1$ show a nonsense mutation, c.814C $>T$ (p.Q272*) on exon 2, as indicated in red, causing premature termination of the SALL1 protein in the mother (patient 2, II: 5) and son (patient 1, III: 1). The pedigree shows the co-segregation of this mutation with Townes-Brocks syndrome phenotypes. 

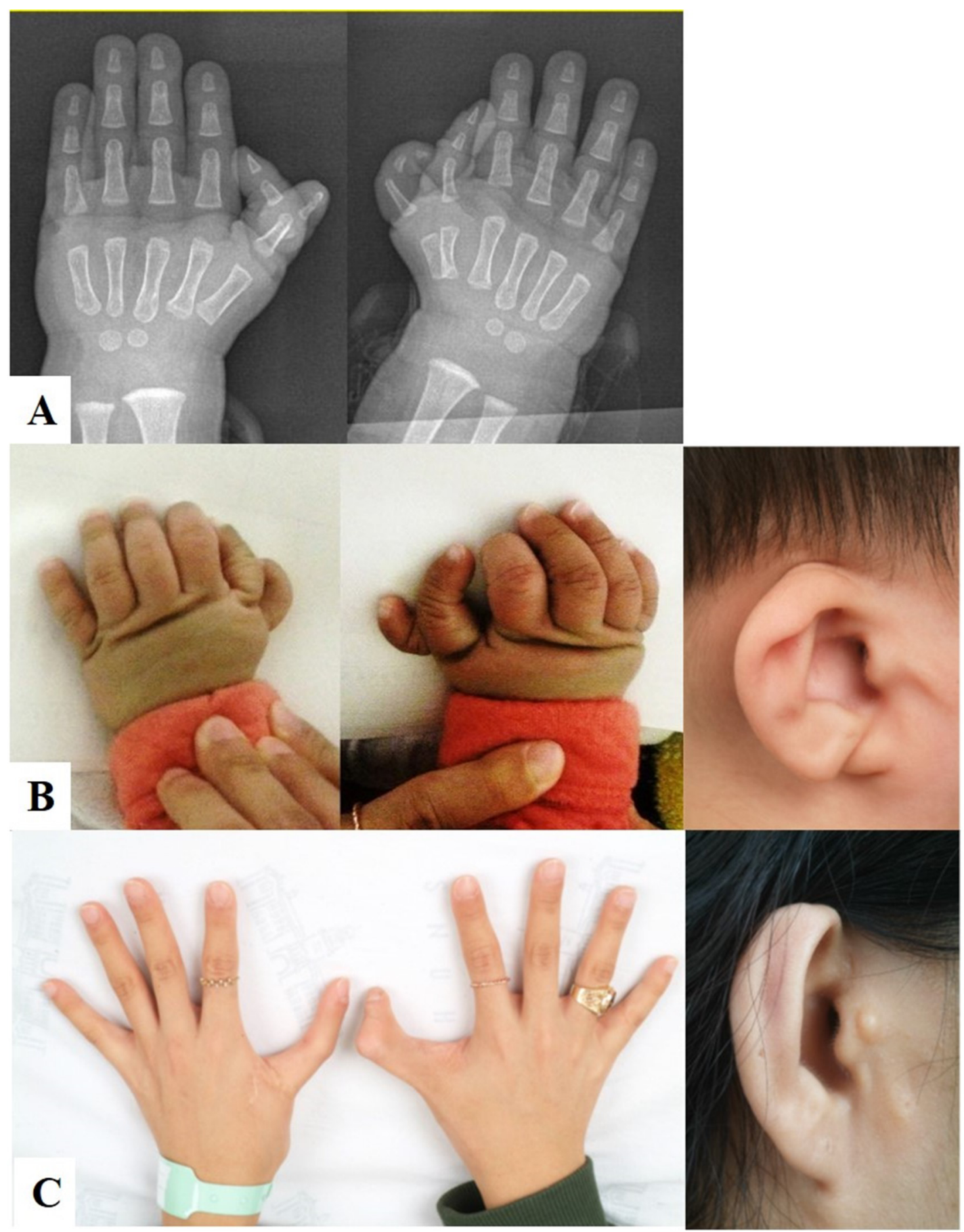

Fig. 2. Radiographs and photographs of patients 1 and 2. The simple radiograph and photograph of the son (patient 1: A, B) show bilateral preaxial polydactyly, triphalangeal thumbs and a small and dysplastic external ear. The photograph of the mother (patient 2: C) shows surgical scars and remnant deformities in both thumbs and her external ear is small and malformed.

suggested bilateral hearing loss; auditory brainstem response threshold was $45 \mathrm{~dB}$ on the right side and $50 \mathrm{~dB}$ on the left. Furthermore, distortion product otoacoustic emission and transiently evoked otoacoustic emission tests yielded no response. The results of laboratory examination showed isolated thyroid stimulating hormone (TSH) elevation without an abnormal free T4 level (TSH $11.39 \mu \mathrm{U} / \mathrm{mL}$, free T4 $1.25 \mathrm{ng} / \mathrm{dL}$ ). However, TSH levels became normalized on the follow-up test (TSH $3.56 \mu \mathrm{U} / \mathrm{mL}$ ). The patient's developmental milestones were grossly normal: he could crawl and say "mama" at eight months, and stand with support at nine months. Surgery for polydactyly was scheduled for 20 months. 


\section{Patient 2 (mother)}

Patient 2 was patient 1 's mother at 31 years of age, and had a history of preaxial polydactyly in both hands and triphalangeal thumbs, which were surgically corrected twice during her childhood (Fig. 2C). She also had bilateral dysplastic ears (Fig. 2C) and bilateral sensorineural hearing loss, requiring hearing aids. However, she did not have an imperforate anus or iris coloboma. She had completed a high school educational course and was of normal intelligence.

\section{Molecular genetic analysis}

The boy and his mother were suspected of having familial TBS based on the medical history and findings of the physical examinations, and molecular genetic analysis for TBS was performed. Written informed consent for the analysis was obtained from the mother. Genomic DNA was isolated from peripheral blood leukocytes. All coding exons and exon-intron boundaries in SALL 1 were amplified using a polymerase chain reaction, and direct sequencing was performed. A nonsense mutation, c.814C >T (p.0272*) in exon 2, was detected in the son. His mother was found to carry the same mutant allele (Fig. 1).

\section{Discussion}

Townes and Brocks [2] first reported a father and five of his seven children who had variable anal, hand, foot, and ear anomalies. Later, reports of similar abnormalities in familial and isolated cases were published and these patients were diagnosed of having TBS [12]. To date, at least 66 cases have been reported worldwide [10]. In 1987, Friedman et al. [13] noted that TBS was associated with a pericentric inversion of chromosome 16. Many molecular analyses had followed and the critical region for TBS was suggested to be located on the distal 1-1.2 Mb of chromosome 16q12.1 [14]. Subsequently, SALL 1 was mapped on $16 q 21.1$ by fluorescence in situ hybridization (FISH) analysis [7] and this gene became a TBS candidate gene, not only because of its chromosomal position, but also because of its structural similarity to the developmental regulator for other genes of the same SALL family [8]. SALL1 is composed of nine $\mathrm{C} 2 \mathrm{H} 2$ protein domains and a $\mathrm{C} 2 \mathrm{HC}$ zinc finger protein domain [7]. SALL1 is expressed in all organs affected by TBS including the kidneys, liver, and fetal and adult brain, suggesting that TBS is most likely a single-gene disorder $[15,16]$.

TBS is a rare genetic disease with an autosomal dominant inheritance pattern; however, the proportion of de novo mutations in all TBS patients is estimated to be up to 50\% [17].
Conventionally, the diagnosis of TBS is based on the presence of the following clinical triad: imperforate anus, dysplastic ear (overfolded superior helices, microtia), and typical thumb malformations (preaxial polydactyly, triphalangeal thumbs, hypoplastic thumbs) without shortening of the radius [1]. However, in addition to the classic triad, other clinical manifestations in the head and neck (hemifacial microsomia), and in ophthalmic (coloboma, microphthalmia, lamellar cataract, or Duane anomaly), auditory (65\% of cases; congenital sensorineural and/or conductive hearing loss), cardiovascular (12-50\% of cases; atrial septal defect, VSD, tetralogy of Fallot, truncus arteriosus, pulmonic valve atresia, or persistent ductus arteriosus), genitourinary (36-42\% of cases; renal agenesis, polycystic kidneys, hypospadia, vaginal atresia with bifid uterus, bifid scrotum, or cryptorchidism), skeletal (52\% of cases; club foot, overlapping toes, syndactyly of toes, missing toes, rib anomalies, or vertebral anomalies), neurologic (10\% of cases; intellectual disability, Arnold-Chiari malformation, cranial nerve palsy, or corpus callosum hypoplasia), and/or endocrine systems (congenital hypothyroidism) can be associated with TBS [17]. Identification of a SALL 1 mutation would confirm the diagnosis of TBS. In our report, the proband (patient 1) presented with an imperforate anus, dysplastic ears with sensorineural hearing loss, preaxial polydactyly with triphalangeal thumbs, and coloboma of the iris and VSD, but the genitourinary, skeletal, and endocrine systems were not affected. Although his mother (patient 2) has the same gene mutation as found in patient 1 , she exhibited only two clinical features (dysplastic ears with sensorineural hearing loss and preaxial polydactyly with triphalangeal thumbs) of the classic triad of TBS.

We found that a p.0272* mutation to be the cause of TBS in this family. p.0272* was reported in a patient whose anomalies included an imperforated anus, triphalangeal bifid thumb, left ear anomaly with hearing loss, facial asymmetry, unilateral renal agenesis with impaired renal function, and 12 pairs of ribs. However, ophthalmic anomalies including iris coloboma and cardiac anomalies were not observed in a previously reported patient with a p.0272* mutation [18]. There have been reports showing wide clinical variations and different levels of severity in TBS, even within one family with the same SALL 1 mutations [19]. Our report supports the highly variable expressivity of TBS.

Clinically, the characteristics of TBS notably overlap with those seen in other syndromes. VACTERL syndrome is the disorder likely to feature most prominently in differential diagnosis. Anal atresia is commonly accompanied by VACTERL-like TBS; however, the frequent presence of vertebral anomalies and 
tracheoesophageal fistulas favors VACTERL syndrome, which has no ear abnormalities or hearing loss. Besides this, BallerGerold syndrome, Goldenhar syndrome, cat eye syndrome, and Holt-Oram syndrome should be excluded when diagnosing TBS $[1,6]$. Therefore, TBS should be considered as a rare causative disorder in the evaluation and differential diagnosis of patients suspected to have multiple malformations.

In this study, we report a Korean family with TBS resulting from a nonsense mutation in SALL 1 who show mild clinical phenotypes. Although TBS is a rare disease, it is inherited in an autosomal dominant pattern, and other familial recurrences have been reported. An accurate diagnosis followed by appropriate management and genetic counseling should be provided to these patients [17].

\section{Acknowledgements}

We thank the patients and their families for participating in this study. This study was supported by a grant of the Korea Health Technology R\&D Project through the Korea Health Industry Development Institute (KHIDI), funded by the Ministry of Health \& Welfare, Republic of Korea (grant number: HI12C0014).

\section{References}

1. Powell CM, Michaelis RC. Townes-Brocks syndrome. J Med Genet 1999;36:89-93.

2. Townes PL, Brocks ER. Hereditary syndrome of imperforate anus with hand, foot, and ear anomalies. J Pediatr 1972;81:321-6.

3. Martínez-Frias ML, Bermejo Sánchez E, Arroyo Carrera I, Pérez Fernández $J \mathrm{~L}$, Pardo Romero $\mathrm{M}$, Burón Martínez $\mathrm{E}$, et al. The Townes-Brocks syndrome in Spain: the epidemiological aspects in a consecutive series of cases. An Esp Pediatr 1999;50:57-60.

4. Cameron TH, Lachiewicz AM, Aylsworth AS. Townes-Brocks syndrome in two mentally retarded youngsters. Am J Med Genet 1991;41:1-4.

5. Ishikiriyama S, Kudoh F, Shimojo N, Iwai J, Inoue T. Townes-Brocks syndrome associated with mental retardation. Am J Med Genet 1996; 61:191-2.

6. O'Callaghan M, Young ID. The Townes-Brocks syndrome. J Med Genet 1990;27:457-61.
7. Kohlhase J, Schuh R, Dowe G, Kühnlein RP, Jäckle $H$, Schroeder $B$, et al. Isolation, characterization, and organ-specific expression of two novel human zinc finger genes related to the Drosophila gene spalt. Genomics 1996;38:291-8.

8. Kohlhase J. SALL1 mutations in Townes-Brocks syndrome and related disorders. Hum Mutat 2000;16:460-6.

9. So HS, Choi HJ, Yoon HS, Hwang JS, Sohn KC. A case of townesbrocks syndrome. J Korean Pediatr Soc 2003;46:382-4.

10. Park SY, Lee WR. Townes-brocks syndrome associated with hypothyroidism in a korean newborn: a case report. J Genet Med 2008;5:136-8.

11. Choi WI, Kim JH, Yoo HW, Oh SH. A family with Townes-Brocks syndrome with congenital hypothyroidism and a novel mutation of the SALL1 gene. Korean J Pediatr 2010;53:1018-21.

12. Monteiro de Pina-Neto J. Phenotypic variability in Townes-Brocks syndrome. Am J Med Genet 1984;18:147-52.

13. Friedman PA, Rao KW, Aylsworth AS. Six patients with the TownesBrocks syndrome including five familial cases and an association with a pericentric inversion of chromosome 16. Am J Hum Genet 1987;41(Suppl):A60.

14. Michaelis RC, Kaiser-Rogers KA, Reitnauer PJ, Rao KW, Powell CM. Refinement of the critical region for Townes-Brocks syndrome. Am J Hum Genet 1996;59(Suppl):A228.

15. Kohlhase J, Wischermann $A$, Reichenbach $H$, Froster $U$, Engel $W$. Mutations in the SALL1 putative transcription factor gene cause Townes-Brocks syndrome. Nat Genet 1998;18:81-3.

16. Kohlhase J, Taschner PE, Burfeind P, Pasche B, Newman B, Blanck C, et al. Molecular analysis of SALL1 mutations in Townes-Brocks syndrome. Am J Hum Genet 1999;64:435-45.

17. Kohlhase J. Townes-Brocks syndrome. In: Pagon RA, Adam MP, Ardinger HH, Wallace SE, Amemiya A, Bean $\sqcup H$, et al., eds. GeneReviews ${ }^{\circledR}$ [Internet]. Seattle, WA: University of Washington, 1993-2015. [http://www.ncbi.n/m.nih.gov/pubmed/20301618]

18. Botzenhart EM, Bartalini G, Blair E, Brady AF, Elmslie F, Chong KL, et al. Townes-Brocks syndrome: twenty novel SALL1 mutations in sporadic and familial cases and refinement of the SALL1 hot spot region. Hum Mutat 2007;28:204-5.

19. Sudo Y, Numakura C, Abe A, Aiba S, Matsunaga A, Hayasaka K. Phenotypic variability in a family with Townes-Brocks syndrome. J Hum Genet 2010;55:550-1. 